the medicine was not a pharmacy item or was not used in a large enough quantity to allow rotation.

Discussion: The equivalent of four times the technician's wage was saved over 12 months. This illustrates striking cost savings gained by efficient, timely rotations and the cost benefits of employing a technician.

Prehosp Disaster Med 2019;34(Suppl. 1):s122-s123

doi:10.1017/S1049023X19002632

\section{Discovering Best Practice Establishing Evacuation Centers for Vulnerable Populations: Findings from Australia and Japan} Dr. Mayumi Kako1, Dr. Malinda Steenkamp $p^{1}$, Dr. Benjamin Ryan ${ }^{2}$, Dr. Paul Arbon ${ }^{1}$, Mr. Yosuke Takada

1. Flinders University, Adelaide, Australia

2. Daniel K. Inouye Asia Pacific Center for Security Studies, Honolulu, United States

3. Okayama University, Okayama, Japan

Introduction: Potentially vulnerable population groups in disasters include the elderly and frail, people who are isolated, and those with chronic diseases, including mental health conditions or mobility issues. The disasters such as the Queensland flood and Great East Japan Disaster in 2011, affected regions of Australia and Japan. This study is followed by two pilot studies in both countries after the disasters. While both countries have different evacuation center procedures for evacuees, the issues regarding the role and responsibility across governments involving planning, setup, and management of evacuation centers demonstrate similarities and differences.

Aim: This paper will report the preliminary findings of a pilot study undertaken with local government officials and humanitarian agencies in Australia and Japan concerning their involvement in planning for, setting up, and managing evacuation centers for vulnerable populations in recent natural disasters. The objective is to illuminate the similarities and differences that officials and agencies faced, and to highlight the resolutions and lessons learned in the preparation of evacuation centers through this event.

Methods: This is the final stage of the study. After completing an analysis of both phases, a comparative framework to highlight similarities and differences was developed.

Results: Each government's role in relation to the establishment of evacuation centers is legally defined in both countries. However, the degree of involvement and communication with non-governmental organizations from the planning cycle to the recovery cycle demonstrates different expectations across governments.

Discussion: While the role of governments is clearly established in both countries based on the legal frameworks, the planning, set-up, and management of evacuation center differs.

Prehosp Disaster Med 2019;34(Suppl. 1):s123

doi:10.1017/S1049023X19002644

Does Medical Presence Decrease the Perceived Risk of Substance-Related Harm at Music Festivals? Dr. Matthew Brendan Munn ${ }^{1}$, Dr. Melissa Sydney White ${ }^{2}$, Dr. Alison Hutton ${ }^{3}$, Dr. Sheila Turris ${ }^{1}$, Haddon Tabb ${ }^{4}$, Dr. Adam Lund ${ }^{1}$, Dr. Jamie Ranse ${ }^{4}$
1. UBC Emergency Medicine, Vancouver, Canada

2. UBC Southern Medical Program, Kelowna, Canada

3. University of Newcastle, Australia

4. UBC Mass Gathering Medicine Interest Group, Canada

5. Menzies Health Institute, Griffith University, Gold Coast, Australia

Introduction: The use of recreational substances is a contributor to the risk of morbidity and mortality at music festivals. One of the aims of onsite medical services is to mitigate substance-related harms. It is known that attendees' perceptions of risk can shape their planned substance use; however, it is unclear how attendees perceive the presence of onsite medical services in evaluating the risk associated with substance use at music festivals.

Methods: A questionnaire was administered to a random sample of attendees entering a multi-day electronic dance music festival.

Results: There were 630 attendees approached and 587 attendees completed the 19 item questionnaire. Many confirmed their intent to use alcohol $(48 \%, \mathrm{n}=280)$, cannabis $(78 \%, \mathrm{n}=453)$, and recreational substances other than alcohol and cannabis (93\%, $\mathrm{n}=541)$ while attending the festival. The majority $(60 \%$, $\mathrm{n}=343$ ) stated they would still have attended the event if there were no onsite medical services available. Some attendees agreed that the absence of medical services would have reduced their intended use of alcohol $(30 \%, n=174)$ and recreational substances other than alcohol and cannabis $(46 \%, \mathrm{n}=266)$.

Discussion: In the context of a music festival, plans for recreational substance use appear to be substantially altered by attendees' knowledge about the presence or absence of onsite medical services. This contradicts our initial hypothesis that medical services are independent of planned substance use and serve solely to reduce any associated harms. Additional exploration and characterization of this phenomenon at various events would further clarify the understanding of perceived risks surrounding substance use and the presence of onsite medical services.

Prehosp Disaster Med 2019;34(Suppl. 1):s123

doi:10.1017/S1049023X19002656

The Effect of Emergency Department Expansion on Emergency Department Patient Flow Prof. Min Joung Kim ${ }^{1}$, Prof. Joon Min Park

1. Department of Emergency Medicine, Yonsei University College of Medicine, Seoul, South Korea

2. Department of Emergency Medicine, Inje University Ilsan Paik Hospital, Goyang, South Korea

Introduction: Overcrowding in the emergency department (ED) has been a global problem for a long time, but it is still not resolved.

Aim: To determine if an ED expansion would be effective in resolving overcrowding.

Methods: This was a retrospective study comparing two 10month periods before (September 2015 to June 2016) and after (September 2017 to June 2018) the ED expansion in an urban tertiary hospital. The existing ED consisted of 45 beds in the adult area and eight beds in the pediatric area. After the construction, the number of beds was not increased, but a fast track 
area was newly established in the adult area, and a 25-bed ward for emergency hospitalized patients was opened.

Results: The number of patients visiting the ED increased from 77,078 to 87,927 . The proportion of patients who returned home without treatment significantly decreased from $11.5 \%$ to $0.9 \%(\mathrm{p}<0.001)$. The number of adult patients increased from 40,814 to 60,720 , but the number of patients who could be treated on the bed decreased (22,166 (54.3\%) vs. $17,776(29.3 \%), p<0.001)$. The number of pediatric patients was similar in both periods. Median ED length of stay (LOS) of total patients increased from $193.0 \mathrm{~min}$ to $205.8 \mathrm{~min}$ ( $\mathrm{p}<0.001)$. Of the 18,900 hospitalized patients during postperiod, 1,255 (6.64\%) were admitted to the emergency ward, and the boarding (from admission decision to hospitalization) time of the admitted patients decreased from $239.2 \mathrm{~min}$ in the pre-period to $190.9 \mathrm{~min}$ in the post-period by $38.3 \mathrm{~min}$. However, more time was required for admission decision in the post-period ( 216.8 vs. $253.3, \mathrm{p}<0.001)$.

Discussion: The ED expansion allowed more patients to be treated, and the emergency ward reduced boarding times of admitted patients. However, due to the increase in the number of patients, the time required for medical treatment increased.

Prehosp Disaster Med 2019;34(Suppl. 1):s123-s124

doi:10.1017/S1049023X19002668

\section{The Effects of Current Cold Chain Management Equipment in Controlling the Temperature of Pharmaceutical Stores in an Australian Defence Force Exercise Environment}

Ms. Liz Daly

The Australian Army, Albury, Australia

Aim: The purpose of this pilot study was to analyze the current cold chain storage methods of Class 8 stores, specifically thermolabile medications and temperature sensitive diagnostics, dressings, and fluids, for the Australian Army in a training area within Australia. This research was designed to identify deficiencies in current storage methods, including the inability to maintain the recommended storage temperature of pharmaceutical stores in accordance with the Therapeutic Goods Administration, as well as foster communication between key stakeholders, including the Royal Australian Army Medical Corps and the Department of Defence Joint Health Command, and to develop a cold chain protocol specific for the Australian Defence Force.

Methods: This pilot study identified the common occurrence of breaches in a specific climate and recommends that current mission essential equipment be replaced. It also discusses the need for clearly defined guidelines with accountability of the stakeholders to ensure that the provision of health support to all Australian Defence Force personnel is in accordance with civilian standards.

Results: This pilot study identified that the carried thermolabile medications and temperature sensitive diagnostics, dressings, and fluids were commonly exposed to temperatures outside the range recommended by the manufacturers. These findings related mainly to the storage equipment for Class 8 stores used by the Army. As a result, it is recommended that such equipment is replaced so that the cold chain storage meets the Therapeutic Goods Administration Guidelines to ensure that health support to Australian Defence Force Personnel in the field is in accordance with the standard of care expected at a civilian health facility.

Discussion: This pilot study has enabled the Australian Defence Force to qualify and quantify the temperature exposure of the medications and stores and engage with key stakeholders to trial and apply new technologies and processes for the management of the cold chain.

Prehosp Disaster Med 2019;34(Suppl. 1):s124

doi:10.1017/S1049023X1900267X

\section{The Efficacy of Blood Lactate on Predicting the Prognosis of Patients with Multiple Trauma \\ Miss Di Hao \\ West China Hospital, Chengdu, China}

Introduction: Because of the rapid progress of multiple trauma patients, the early mortality rate is high. Therefore, early assessment of the severity and prognosis of multiple injuries is crucial for timely treatment and improvement of prognosis. So we need to find parameters related to mortality and severity of multiple trauma.

Aim: To find out parameters related to mortality and severity of multiple trauma.

Methods: This was a single center, trauma registry based, observational cohort study. Data were collected from consecutive patients with multiple trauma who presented to the emergency department of a tertiary referral hospital between April 2015 and December 2016. The main outcomes studied were 28-day in-hospital mortality, 24-hours mortality, emergency operation rate, and ICU admission rate.

Results: 444 patients were eventually included in the study, including 337 males (75.9\%) and 107 females (24.1\%). The 28-day survival group consisted of 381 patients (85.8\%) and the death group accounted for the other 14.2\%. Multivariate logistic regression analysis showed that heart rate, peripheral oxygen saturation, lactic acid, partial pressure of carbon dioxide, plasma albumin, hematocrit, and Glasgow score were independent risk factors for 28-day mortality. The area under the ROC curve (AUC) of the above indicators was $0.669,0.547,0.868$, $0.512,0.740,0.627$, and 0.815 , and the AUC value of lactate was the maximum.

Discussion: When the body suffers from severe trauma, it loses a lot of blood and reduces the circulating blood volume, which leads to absolutely insufficient hemoglobin content and hypoxia of tissue cells. The plasma lactate content increases at this time. Therefore, lactate can be used as an important prognostic parameter for patients with multiple trauma. In addition, we can use lactate to revise the existing trauma score to enhance its effectiveness. Prehosp Disaster Med 2019;34(Suppl. 1):s124 doi:10.1017/S1049023X19002681 